\title{
La gestión anacrónica de los centros educativos en Costa Rica
}

\author{
Out-Dated Management Styles In Costa Rican Schools
}

\author{
Bayron Cortés Rodríguez' \\ Universidad de Costa Rica \\ San José, Costa Rica \\ bayron.cortesrodriguez@ucr.ac.cr
}

Recibido 16 junio 2015 • Aceptado 10 febrero 2016 • Corregido 30 marzo 2016

\begin{abstract}
Resumen. El presente artículo expone la necesidad de cambio en el sistema educativo actual de Costa Rica, el modelo de gestión tradicional frente a las nuevas estrategias de gestión. Para ello, se ha analizado la forma tradicional de administración de los centros educativos, con el propósito de conocer cómo se puede adaptar la gestión educativa a las exigencias de la sociedad del siglo XXI. El presente artículo se ha divido en cuatro áreas: a) Contextualización; b) Modelo anacrónico de la gestión de centros educativos; c) El sistema educativo costarricense; d) Propuesta de gestión.
\end{abstract}

Palabras clave. Sistema anacrónico de gestión; administración educativa; estrategias de gestión; crisis en la forma de gestión

\begin{abstract}
This article discusses the need for changes to occur in the traditional management model of the Costa Rican school system and juxtaposes it against new management strategies. Traditional styles of school administration are analyzed with the objective of adapting them in order to fit the demands of the XXIst century. The article is divided into four sections:: a) Contextualization; b) Outdated management model Educational Centers; $c$ ) The Costa Rican education system; d) Proposal management.
\end{abstract}

Keywords. System anachronistic management; educational administration; management strategies; crisis management mode

\section{Introducción}

La sociedad como tal, es un sistema complejo sometido a procesos constantes de cambio en las diversas esferas que lo componen; la evolución y actualización del ser humano, producto de la dinámica global y la aceleración de los procesos de cambio, lo hacen aventurarse en posibles lagunas del saber y del conocimiento. El sistema de gestión de nuestros centros educativos se encuentra inmerso en la problemática de desactualización, lo que no le permite mantener un ritmo que responda a las necesidades de la sociedad actual.

1 Master en Administración Educativa, Universidad de Costa Rica. Profesor de Posgrado en la Maestría en Administración Educativa, Universidad de Costa Rica. Licenciado en Educación General Básica I y II Ciclo, Universidad Católica de Costa Rica. 
Se ha asumido una forma tradicional, obsoleta y estática de gestionar nuestros centros educativos; se gestiona en forma anacrónica, por lo que se habla de una crisis en la manera de administrar las instituciones, olvidándonos de las demandas y exigencias de la sociedad. Así, se encuentran centros educativos sin una doctrina institucional definida, sin objetivos, sin una visión y una misión; instituciones educativas agotadas y sin una identidad propia.

La gestión educativa, la define Weber, citado por Dávila (2001), dentro de la Teoría Burocrática, como "una forma de organización humana basada en la racionalidad" (p. 134); es decir, en la búsqueda de mecanismos que permitan el logro de las expectativas planteadas. En un sentido más amplio, es la eficiencia en la toma de decisiones basadas en lo que se debe hacer y expresarlo en el funcionamiento institucional, mediante un canal de comunicación claramente definido. No obstante, no consiste en la continuidad de procedimientos que favorezcan una estandarización; sino por el contrario, es adaptar y garantizar en la institución, un modelo moderno de gestión, contemplando las exigencias competitivas del quehacer educativo.

Para Punset (2011), "la educación actual penaliza el error y la equivocación cuando es a partir del error que podemos sacar las mejores enseñanzas siendo un proceso positivo dentro del propio aprendizaje del alumno" (p. 1). La figura del docente resulta importante en el proceso de enseñanza aprendizaje, como así mismo, la función del gestor resulta determinante en potencializar un sistema innovador.

La gestión tradicional entiende a la administración como una serie de disposiciones atendidas, la presentación de formularios o informes y la propuesta de planes o ejecución sin un fin eficiente. Las instituciones educativas requieren un modelo acorde con las necesidades sociales, personales y tecnológicas. Una administración de calidad, adaptables a las nuevas exigencias de la sociedad.

\section{Contextualización}

La globalización comprende un fenómeno de nuestra sociedad; se ha convertido en un flagelo que penetra la esencia de nuestra organización transformando con su paso el Estado y por ende, la cultura, la educación y los fines que persiguen nuestra organización social. La educación es la herramienta fundamental que debe formar a la persona ante esos cambios holísticos globales y que, a la vez, le permita adaptarse y ser parte del progreso de una nación.

Medina y Ortegón (2006) describen una serie de características de la globalización, entre ellas se mencionan las siguientes:

- Se refleja como un macro fenómeno que abarca a una extensión geográfica propia de la interacción social, es decir, que la globalización se expande a las diferentes sociedades que genera las mismas tecnologías de la comunicación y del transporte.

- Se expande a grandes velocidades de las comunicaciones virtuales. 
- Abarca las realidades sociales, así como las actividades de producción y de consumo.

- Comprende las interacciones económicas y la de los mercados.

- Se da la uniformidad de ideas y de las prácticas políticas.

- Presenta símbolos comunes de una cultura global, como son las marcas comerciales que se presenta de manera universal.

- Implica impactos ambientales. (p. 87)

La transformación del Estado ha tipificado políticas educativas que no responden al interés de los estudiantes; las perspectivas de los mismos son muy distintas a las expectativas de nuestros antepasados. Para Prensky, citado por Calleja (2010): "el sistema educativo está planeado como una pirámide, estando los administrativos arriba en la cúspide de la pirámide, los profesores intermedios en el centro de la pirámide y los estudiantes abajo en la base de la pirámide" (párr. 7).

Es preciso cambiar este método, propio del siglo XIX, por uno más asociativo, donde alumnos, profesores y administrativos establezcan alianzas y se escuchen mutuamente. Asimismo, se destaca que "el papel del docente es preparar a los estudiantes para el futuro, para que descubran su talento" (Larrañaga, 2012, p. 8). Las generaciones actuales aprenden de manera muy distinta; el uso del internet y los medios electrónicos les permiten ingresar a un mundo de conocimiento que, comparados con nuestra educación actual, no responden a sus expectativas e intereses, permeando la educación formal y tornándola poco atractiva. Antes de llegar a las aulas han adquirido un aprendizaje previo e incluso actualizado.

En palabras de Robinson (2006), el sistema educativo fue diseñado, concebido y estructurado para una época diferente. El sistema educativo actual sigue basándose en estructuras ideológicas heredadas de siglos anteriores. Las instituciones educativas deben ser transformadoras para que se den circunstancias en las cuales surjan los talentos personales de los estudiantes como fin último de nuestra labor; devolver la pasión, el entusiasmo, y la dedicación a nuestros estudiantes.

\section{Los sistemas educativos anacrónicos}

Los sistemas anacrónicos constituyen sistemas arcaicos que han viciado algunas sociedades, impidiéndoles su pleno desarrollo, generando una evolución lenta e incapaz de transformarse y adecuarse a las demandas de los sistemas sociales.

Así mismo, Robinson (2006) manifiesta que las sociedades que han tenido poco crecimiento económico o no se han desarrollado integralmente, son sociedades estancadas, el pasado sigue introduciéndose en su presente y en su futuro como única opción de crecimiento; son sociedades anacrónicas, viven del pasado. Los centros educativos no escapan de esta 
problemática, la manera más sensata de preparar a los estudiantes es con los conocimientos y necesidades del pasado; personas poco competitivas en un mundo globalizado. De esta manera, la vida educativa es un espejo del pasado; reglamentos casi militares, falta de individualización en la capacidad del estudiante, riguroso sistema de aulas, grupos, grados y títulos, así como el papel autoritario del docente, son elementos de mediocridad y adaptación social.

Para Punset (2011), "tenemos los mismos sistemas educativos que hace cincuenta años cuando lo que imperaba era una sociedad industrial mientras que la sociedad actual está enfocada a los servicios y la información" (p. 1). Lo que ocurre en la actualidad con la educación, incluso en los llamados colegios de calidad, es lamentablemente anacrónico. Los padres confían en matricular en un sistema educativo que preparara a sus hijos para la vida del futuro, así como los maestros advierten que la falta de educación destruiría las oportunidades del estudiante en el mundo del mañana, con una educación del pasado. Son inconsistentes los discursos de los sistemas educativos anacrónicos.

Como dice Prensky (2011): “Debemos aprender cuáles son las verdaderas necesidades de los alumnos, escucharlos y conocer sus intereses y pasiones, sólo así lograremos prepararlos para enfrentar un mundo donde los cambios son cada vez más rápidos" (párr. 5). Debemos procurar desarrollar sistemas educativos más acordes con las necesidades de nuestros educandos, propiciando un aprendizaje más significativo, innovador y creativo; solo de esta manera estaremos desarrollando una evolución basados en las necesidades e intereses de los estudiantes, creando una conciencia de sociedad que se transforma, evoluciona y cambia constantemente.

\section{El sistema educativo costarricense}

Elsistemaeducativo costarricense, a través de la historia, ha sido reflejo dela implementación de sistemas anacrónicos en donde se estructura un método de enseñanza con características específicas, siendo las aulas recintos académicos con clases magistrales que vierten en los estudiantes conocimientos que deben interiorizar de forma memorística, tratando de crear máquinas que actúan mecánicamente coartando el desarrollo integral del conocimiento; lo que ocasiona, en los actores, un desinterés por la educación formal, impidiendo el nacimiento de ideas innovadoras y el desarrollo de las potencialidades que todo ser humano trae consigo.

Es necesario mantener un ritmo de desarrollo acorde con las necesidades actuales; las nuevas teorías de la educación deben proyectarse de forma que respondan al desarrollo acelerado de nuestra sociedad.

El nuevo siglo cuestiona los procesos educativos y hace reflexionar, constantemente, sobre las funciones que deben cumplir los educadores y el sistema educativo; señala y reta al campo pedagógico sobre lo que es y debe ser la educación y cómo se debe dar el proceso de enseñanza aprendizaje y el manejo de la información. 
Por otra parte, la globalización es el resultado de la implementación del conocimiento y el desarrollo de los medios masivos de información, por lo que la educación debería ser un medio, a través del cual se esté a la vanguardia de los procesos de cambio de la humanidad.

El sistema educativo costarricense es una paradoja, en el tanto que contempla un desarrollo integral del ser humano pero, a su vez, implementa políticas educativas, programas de estudios y metodologías de aprendizaje que no responden a las necesidades actuales que demandan un mundo en constante evolución.

El relanzamiento y reestructuración del actual sistema educativo costarricense, planea tópicos como: la educación rural, el fortalecimiento de la educación técnica, la calidad de la educación de la educación, la igualdad de género, ente otros. Sin embargo, todos estos requieren de un esfuerzo constante y compromiso por parte de todos los participantes del proceso, con el fin de alcanzar las expectativas plasmadas en las fuentes escritas, las cuales se convertirán en letra muerta si los actores del proceso no le dan vida al mismo para eliminar las desigualdades que inmovilizan la inserción, permanencia y el éxito en los sistemas educativos actuales.

El Ministerio de Educación Pública (MEP, 2012), en su programa de Calidad de la Educación, manifiesta debilidades y fortalezas del sistema educativo costarricense:

Debilidad: Subsiste una extensa red de centros de enseñanza que operan en instalaciones ruinosas y muestran bajas promociones, en la que se mantienen altos niveles de exclusión de estudiantes en séptimo año, una baja cobertura de la educación diversificada y amplias brechas territoriales y sociales en el logro educativo.

Fortaleza: El promedio general de exclusión educativa se redujo en 2012, pero se mantiene por encima del $16 \%$ en séptimo año.

Debilidad: Existe una educación superior desarticulada, con muchas universidades privadas en torno a las cuales se desconocen hasta los datos más elementales sobre su desempeño.

Fortaleza: El porcentaje de personas de 25 años y más que tienen un grado universitario se cuadruplicó en casi cuatro décadas. No obstante, debido a los cambios en la estructura por edades de la población, hoy este grupo no llega a representar la cuarta parte de las personas de ese rango etario (22,6\%).

Debilidad: El Ministerio de Educación Pública (MEP) arrastra serios problemas en materia de contratación docente y sigue creciendo la sobreoferta de carreras de Educación, la mayoría de ellas sin acreditar. (p. 10)

A pesar de los esfuerzos del sistema educativo costarricense, aún queda mucho por mejorar. El programa de la Calidad de la Educación manifiesta múltiples debilidades de nuestro sistema educativo, ya que no se puede garantizar una educación de este tipo cuando la deserción en los estudiantes de séptimo año es un problema aun sin solucionar, por ejemplo. 
Así mismo, el sistema educativo costarricense es excluyente, puesto que solo un porcentaje poco significativo logra la obtención un título universitario y no existen datos o regulaciones de lo que están produciendo las universidades privadas. Son estas inconsistencias las que determinan el gobierno anacrónico de nuestro sistema educativo.

Al respecto, Robinson (2006) sostiene que cualquier estudiante puede seguir un programa de estudios inadecuado, sea cual sea la vía de formación (internet, presencial o a distancia). Lo necesario en el presente caso, es crear mecanismos de defensa en los estudiantes que les permitan tomar decisiones asertivas y descartar todo aquello que los medios tecnológicos no constituyan un beneficio para el desarrollo y estructuración de sus potencialidades. No obstante, cabe resaltar que sin profesores comprometidos, que guíen y estimulen a los estudiantes, continuaremos con los actuales problemas y deficiencias educativas.

Resultaevidentequelaadministración dela educaciónyporende, lostrabajadores delaeducación costarricense, deben adquirir prioritariamente un compromiso con las directrices, lineamientos y normativas vigentes, con el fin de lograr un sistema educativo más acorde con las necesidades reales y menos enajenadas. Lograr este compromiso representa uno de los retos más importantes para todos aquellos gestores que busquen que los centros escolares trasciendan con éxito.

De acuerdo con las teorías administrativas vigentes, las organizaciones educativas deben replantearse, ajustarse a los cambios sociales y a las necesidades educativas vigentes, apropiándose de nuevos esquemas de participación, jerarquización y compromiso; significa desarrollar un nuevo aprendizaje que propicie todos estos componentes en relación con el compromiso organizacional:

... ya no se trabaja individualmente, ahora las relaciones son grupales; antes se trabajaba bajo el parámetro de autoridad-obediencia, ahora existe la confianza entre los miembros de un grupo; antes se delegaba autoridad, ahora existe la interdependencia y la responsabilidad compartida. (Mena, 2009, p. 14)

Es importante destacar que el administrador del centro educativo, debe reconocer que en la inclusión se hace necesaria la actualización, el cambio y el compromiso, pues todo el equipo de trabajo debe saber que cada miembro de la comunidad es único y, por lo tanto, no puede ser comparado con nada ni con nadie; asumir que el reto está en la diversidad, que solo con la mentalidad abierta al cambio se pueden lograr oportunidades de ser innovadores. Su propósito deberá ser la creación de espacios donde se fomente la igualdad de derechos y oportunidades, el fortalecimiento de una educación de calidad, a través del reconocimiento de la equidad.

\section{La gestión educativa y el mejoramiento de la educación}

Las necesidades que demanda el mundo en la actualidad y la globalización, afectan directamente la manera como se desarrolla la gestión educativa; la obligatoriedad de emprender 
eficaces y eficientes transformaciones enfrentan importantes desafíos para dar respuestas, oportunas y pertinentes a las nuevas necesidades de los educandos.

El establecimiento de una gestión oportuna y un liderazgo empoderado en el sistema educativo costarricense, que se responsabilice por implementar directrices anuentes al mejoramiento de la calidad de la educación, son necesarias en la implementación de acciones en cuanto a la formación y actualización de los docentes, consolidando y orientando a los estudiantes en el desarrollo de sus potencialidades y habilidades, formando ciudadanos que logren, mediante la institución, competencias básicas para el logro de los objetivos y metas individuales.

El mejoramiento en la educación requiere de un compromiso constante que fortalezca la igualdad de oportunidades y el desarrollo de competencias individuales de dicha población. La gestión educativa se constituye de procesos organizados que proyectan el logro de los objetivos y metas planteadas, a corto y mediano plazo. Una gestión efectiva requerirá de planificación, implementación,fiscalizaciónyevaluación delos procesosqueconduzcanalaobtenciónderesultados prácticos, acordes con la evolución e inserción en el mundo actual. Una gestión educativa efectiva es la clave para el éxito y permanencia de los participantes de este gran proyecto. Es fundamental lograr que todos se involucren para lograr lo que se quiere y así, mejorar permanentemente.

Los centros educativos se han transformado, pasando de instituciones de conceptos y políticas cerradas e individualistas, a sistemas más amplios y estructurados, lo que requiere de una gestión educativa cuya planificación demanda un mayor compromiso con nuevas formas de gestión que permitan el cumplimiento de las nuevas estructuras organizacionales, capaces del desarrollo de potencialidades e igualdades en el sistema educativo, dividida en cuatro ámbitos, a saber: gestión directiva; gestión pedagógica y académica; gestión administrativa y financiera; y gestión de la comunidad.
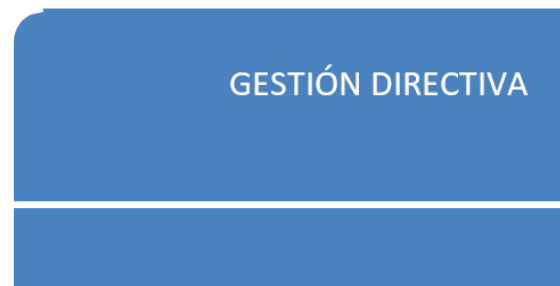

GESTIÓN ADMINISTRATIVA Y FINANCIERA
GESTIÓN PEDAGÓGICA Y ACADÉMICA

GESTIÓN EDUCATIVA

\section{Figura 1. Gestión educativa}

Nota: Elaboración propia, 2015. 
El centro educativo no solo es un espacio donde se adquiere la educación formal; debe ser un lugar donde se propicien espacios de formación integral mediante la participación y convivencia de los diferentes actores en los diversos ambientes de interrelación. Es también, un lugar en el que, además del aprendizaje académico y el desarrollo de habilidades, se fortalecen aspectos de la personalidad como la convivencia, la amistad, la práctica de valores personales y sociales que permitan la construcción de estructuras de la personalidad que permitan a los estudiantes mayores oportunidades de proyección social. Asimismo, es en el centro educativo donde se materializa el currículo, se desarrolla el plan de estudios y las actividades pedagógicas.

Para el logro de las metas institucionales, es necesario contar con herramientas que permitan el logro de los objetivos establecidos; entre estas, se pueden contemplar el plan de estudios y el mejoramiento de la calidad de la educación. Estos propósitos, metas y acciones pertinentes son la identidad o doctrina institucional, la cual debe ser conocida por todos para lograr el compromiso de cada uno, bajo el liderazgo del gestor educativo y su equipo de trabajo.

En el centro educativo se deben establecer cuatro fases para garantizar una gestión de calidad.

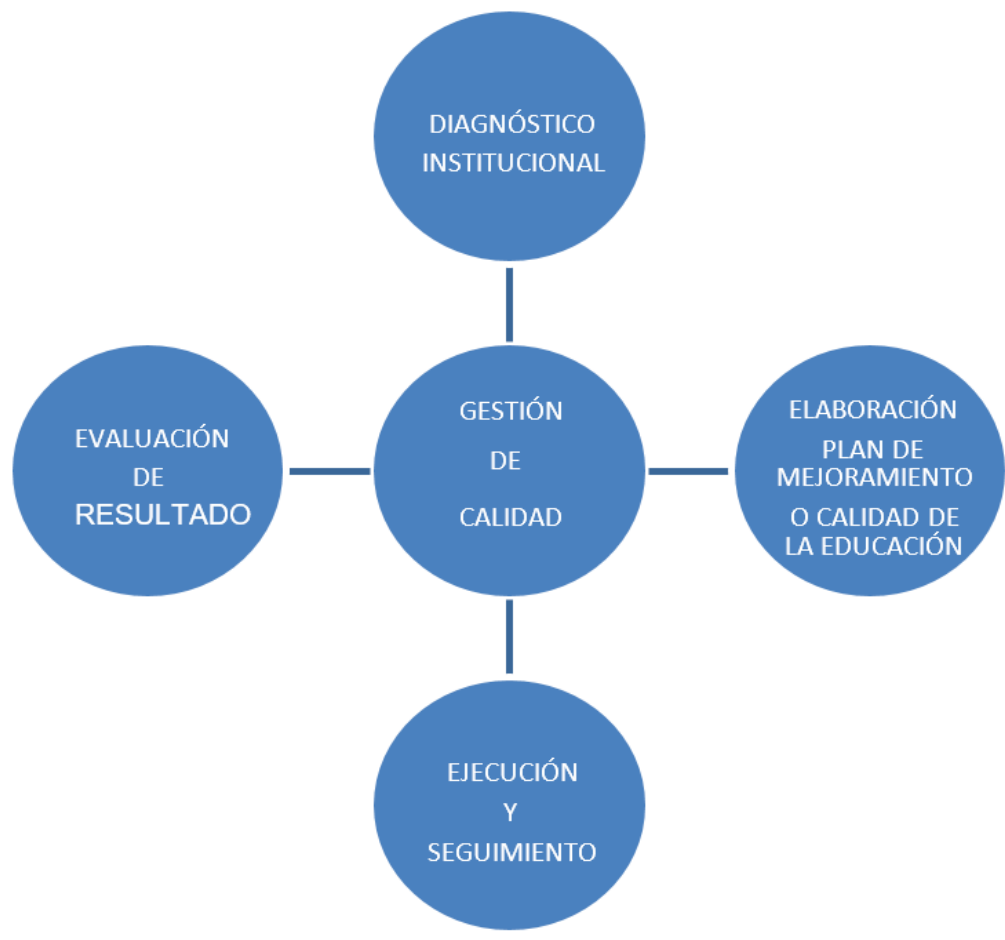

Figura 2. Gestión de Calidad

Nota: Elaboración propia, 2015. 
Las Instituciones que desarrollan acciones para el mejoramiento de la calidad de la educación, defieren en cuanto a la necesidad de implementar las siguientes características:

- Saben hacia dónde van. Cuentan con un conocido y consensuado proceso educativo, con principios y fundamentos orientados en su quehacer; estos principios se concentran en una identidad institucional y una doctrina institucional.

- Establecen los lineamientos del Ministerio de Educación Pública, acerca de lo que los estudiantes deben saber y saber hacer.

- Establecen la efectividad del tiempo escolar y la utilización de sus recursos.

- Tienen un modelo organizativo basado en el liderazgo claro del gestor y en el trabajo en equipo.

- Apoyan y aprovechan el talento de sus estudiantes, brindándoles apoyo y espacios necesarios para desenvolvimiento de habilidades.

- Ofrecen oportunidades para que los estudiantes aprendan con interés y motivación.

- Tienen altas expectativas sobre las capacidades de sus estudiantes.

- Construyen ambientes adecuados de interacción entre estudiantes y docentes.

- Usan el tiempo adecuadamente, haciendo una rigurosa programación en todas las actividades que se realicen durante el año escolar.

- Ofrecen espacios de convivencia y promueven el desarrollo personal y social.

- Articulan acciones con la comunidad y padres de familia.

- Disponen de programas complementarios, garantizando el éxito escolar.

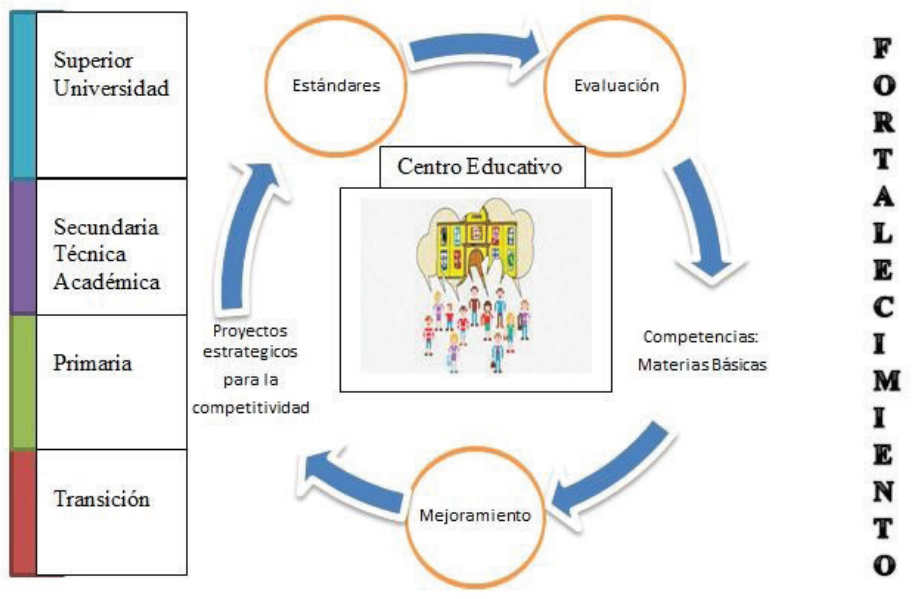

Figura 3. Competencias para el mejoramiento continuo

Nota: Elaboración propia, 2015. 


\title{
Conclusiones
}

\author{
A continuación, la Tabla 1 presenta las principales conclusiones sobre el tema, en función \\ de una propuesta de gestión.
}

\section{Tabla 1}

\section{Cuadro comparativo entre el sistema anacrónico (Robinson, 2006) y el sistema de gestión (propuesta)}

\begin{tabular}{l} 
Sistema Anacrónico - Robinson \\
\hline El sistema educativo costarricense no \\
está exento del sistema anacrónico \\
de la educación; el tipo de enseñanza \\
repetitiva y de memorización \\
fomenta en los estudiantes la \\
pérdida del interés por estudiar y \\
disminuya el interés por generar \\
ideas, ser innovadores, adquirir y \\
reconocer las propias habilidades, \\
talentos e intereses personales
\end{tabular}

El mejoramiento se basa en el cumplimiento de un plan de estudios y políticas institucionales generalizadas

Un estudiante puede llevar un programa de estudios inadecuado, en cualquier modalidad (en línea, presencial, a distancia)

Es en el centro educativo donde se gesta el proceso de enseñanza y aprendizaje

Los centros educativos son instituciones cerradas y aisladas a funcionar para cumplir sus propósitos, desarrollar y consolidar su estructura organizacional.

Existe una figura autoritaria de liderazgo, con una sola vía de comunicación, encargada de la toma de decisiones, autócrata y auto evaluativo.

\section{Sistema de Gestión - Propuesta}

El nuevo siglo cuestiona los procesos educativos y hace reflexionar constantemente sobre las funciones que deben cumplir los educadores y el sistema educativo; señala y reta al campo pedagógico sobre lo que es y debe ser la educación, y como se debe de dar el proceso de enseñanza-aprendizaje y el manejo de la información La globalización es otro aspecto producto del impacto de la sociedad del conocimiento y la información. El mundo se ha convertido en una aldea global y la educación se convierte así, en un bien necesario para las personas

El sistema educativo costarricense apunta hacia el desarrollo integral del ser humano, en un plano de igualdad de oportunidades plantea nuevos programas de estudio, fortaleciendo aspectos conceptuales y metodológicos, buscando reformular el proceso de formación de valores. Además, da énfasis a una visión de largo plazo, postulando una relación intrínseca entre la familia y el sistema educativo

El mejoramiento requiere que se conforme un sistema que asegure una calidad de la educación que esté orientada al fortalecimiento de las igualdades educativas y al desarrollo de las potencialidades de los estudiantes en todos los ámbitos y especialidades que permitan el desarrollo de sus destrezas y habilidades

Lo importante es establecer una buena orientación para que los esfuerzos se enfoquen en la dirección correcta y así, sacar lo mejor de cada uno

La tecnología es muy útil, pero sin buenos profesores que animen e involucren a sus estudiantes, seguiremos igual. Lo importante es establecer una buena orientación para que los esfuerzos se enfoquen en la dirección correcta y así, sacar lo mejor de cada uno

El centro educativo debe ser un espacio de convivencia y aprendizaje formal pero integral, debe ser el resultado de la interacción de diversos factores que establezcan lazos de convivencia, amistad y valores que permitan la construcción de estructuras en el desarrollo de las potencialidades de los participantes

Los centros educativos se encuentran inmersos en un proceso de transformación, creando una apertura para el aprendizaje global mediante superestructuras organizacionales y administrativas que cumplan con los parámetros establecidos con el propósito de desarrollar las capacidades y las potencialidades de los estudiantes

Existe un continuo fortalecimiento de la gestión y liderazgo en función de la educación, así como la implementación de mecanismos adecuados que permitan un debido proceso de inducción, selección, formación y evaluación del desempeño, necesarios para el mejoramiento de la calidad de la educación

Nota: Elaboración propia (2015), a partir Robinson (2006). 


\section{Referencias}

Calleja, R. (2010). Un modelo educativo para el siglo XXI. Comunidad escolar, 88. Recuperado de: http://comunidad-escolar.pntic.mec.es/880/report1.html

Dávila, C. (2001). Teorías organizacionales y administración. México: McGraw-Hill Interamericana. Larrañaga, A. (2012). El modelo educativo tradicional frente a las nuevas estrategias de aprendizaje. Universidad de La Rioja. Recuperado de: http://reunir.unir.net/handle/123456789/614

Medina, J. y Ortegón, E. (2006). Manual de prospectiva y decisión estratégica: bases teóricas e instrumentos para América Latina y el Caribe. Chile: Editorial Naciones Unidas.

Mena, O. (2009). Gerencia del futuro. San José, Costa Rica: Universidad de Costa Rica.

Ministerio de Educación Pública (MEP). (2012). El centro educativo de calidad como eje de la educación costarricense. San José, Costa Rica: MEP.

Prensky, M. (2011). El sistema educativo anacrónico. [Archivo de video]. Recuperado de: http:// www.rtve.es/alacarta/videos/redes/redes-sistema-educativo-anacronico/1044110/

Punset, E. (2011). El sistema educativo anacrónico. [Archivo de video]. Recuperado de: http:// www.rtve.es/television/20110304/redes-sistema-educativo-anacronico/413516.shtml

Robinson, K. (2006) Las escuelas matan la creatividad. [Archivo de video]. Recuperado de: https:// www.youtube.com/watch?v=nPB-41q97zg 